\title{
An $n$-fold Coincidence Problem in Physiology
}

\author{
M. TEN Hoopen AND H. A. Reuver \\ Institute of Medical Physics TNO, Utrecht, The Netherlands \\ (Received 4 September 1964, and in revised form 28 December 1964) \\ An approximate solution is given for a problem encountered in the field \\ of psychophysics and theoretical neurophysiology: the time sequence of \\ events that occur if a minimal number of Poisson distributed identical items \\ (e.g. light quanta or nervous stimuli) arrive within a certain time interval.
}

\section{Introduction}

This paper deals with a queuing problem which is in general terms defined as follows. Events occurring at random, as a function of time for instance, have an effect that is equal for each event. Asked is the probability that within a certain time interval once or more than once a given number of effects is present.

This problem originates, among others, from the field of psychophysics, viz. from the theory (Bouman \& Van der Velden, 1947) presuming that the perception of a small and short light flash of about threshold intensity depends on a minimal number of light quanta $k$ effectively absorbed by the visual purple within a summation interval and within a small area of the retina. It is assumed that the quanta arrive according to a Poisson process with parameter $\mu$ and that only temporal and no spatial summation is at stake. The probability of perception during an exposure time $t$ is called $W(t)$. A related quantity of interest is $(\mu t)_{0.6}$, the value of $\mu t$ for which the probability of perception $W(t)=0.6$.

Formally this model is equivalent to one encountered in theoretical neurophysiology and posed by Rapoport (1950). Consider a formalized neuron receiving a shower of stimuli, identical in effect and completely randomized in time with an average frequency $\mu$, and responding to the $k$ th stimulus of a group which falls within the period of latent summation $\tau$, provided none of the $k$ stimuli impinges during the absolute refractory period lasting $r$ units of time.

Only an absolute refractory period is taken into account during which previous and new effects are neglected. The probability density function of 
the time interval between successive firings is wanted, which is, except for a retardation of duration $r$, equal to the time derivative of $W(t)$, denoted by $p(t)$.

In the following the summation time $(\tau)$ will be called the holding time of the items.

An exact solution has been given by Van der Velden (1944) for $k=2$ and for larger values of $k$ for $t \leq \tau$. For $k=3 W(t)$ has been obtained by Van Elteren \& Gerrits (1961) from a set of recurrence relations and computed for $t / \tau$ up to 4 . This procedure is in principle extendable to any $t / \tau$, but as the authors remark in practice feasible for small values only. In contrast with this, the approximation here presented results in a fairly compact form if expressed in the Laplace transform notation.

If these models are correct, a question we will not enter into at the moment, a means to compute the probability density function in the neuron model case for higher values of $k$ seems to be of interest. For a neuron to fire, the number of stimuli $k$ required within a few milliseconds has been suggested to be about 10 (Eccles, 1953), but this number is probably much higher (Sholl, 1956). Equally, values of $W(t)$ or $(\mu t)_{0.6}$ for $k>3$ and $t / \tau>4$ are useful in the present status of the quantum theory in vision. Whereas the right value of $k$ is still a matter of discussion, the estimates range mostly from two up to eight; occasionally even larger values are claimed.

We must admit that we have no estimate of the error of the approximation. Therefore, at present, we have to limit ourselves to comparison with known results from the exact solution as well as with Monte Carlo simulations.

\section{An Approximation}

Adopting the nomenclature of the neuron model case for a moment the approximation is elaborated as follows. $p(t)$ is defined as the probability density function of the interval between the moment that a neuron leaves the refractory period and the next firing. $W(t)$ is defined as the distribution function of the same interval. $P_{h}(t)$ is the probability that a neuron is storing $h(<k)$ effects of stimuli at time $t$ after leaving the last refractory state at time $t=0$. The arrival of stimuli being Poisson distributed the probability of arrival of a stimulus in $(t, t+\mathrm{d} t)$ is equal to $\mu . \mathrm{d} t$, whereas the probability of arrival of two or more stimuli in $(t, t+\mathrm{d} t)$ is of second or higher order in $\mathrm{d} t$ and thus vanishes. The probability of extinction of the effect of a stimulus in $(t, t+\mathrm{d} t)$ when there are $h$ effects present and no reaction has yet occurred is set equal to $\eta_{h} . \mathrm{d} t ; \eta_{h}$ being dependent on the past history. For $t<\tau, \eta_{h}$ is equal to zero. For $t>\tau$, when it is assumed that the moments of arrival of stimuli are nearly homogeneously distributed over the interval $(t-\tau, t), \eta_{h}$ is approximated by $h / \tau$. In the terminology of stochastic processes 
this amounts to a Markovization of a process which is essentially nonMarkovian. Although we have no rationale for this expression it is intuitively clear that the probability of extinction is larger the larger the number $h$ of the stimuli summated and the shorter the holding time.

Again, the probability that two or more stimuli extinct in $(t, t+\mathrm{d} t)$ vanishes.

Then we have:

$$
\begin{aligned}
P_{h}(t+\mathrm{d} t)=P_{h-1}(t) & \times \text { probability of arrival of a stimulus in }(t, t+\mathrm{d} t) \\
& \times \text { probability of no extinction in }(t, t+\mathrm{d} t) \\
+\quad P_{h}(t) & \times \text { probability of arrival of a stimulus in }(t, t+\mathrm{d} t) \\
& \times \text { probability of extinction in }(t, t+\mathrm{d} t) \\
+\quad P_{h}(t) & \times \text { probability of no arrival of a stimulus in }(t, t+\mathrm{d} t) \\
& \times \text { probability of no extinction in }(t, t+\mathrm{d} t) \\
+P_{h+1}(t) & \times \text { probability of no arrival of a stimulus in }(t, t+\mathrm{d} t) \\
& \times \text { probability of extinction in }(t, t+\mathrm{d} t) .
\end{aligned}
$$

Further,

$$
\begin{aligned}
p(t), \mathrm{d} t=P_{k-1}(t) & \times \text { probability of arrival of a stimulus in }(t, t+\mathrm{d} t) \\
& \times \text { probability of no extinction of a stimulus in }(t, t+\mathrm{d} t) .
\end{aligned}
$$

Letting $\mathrm{d} t \rightarrow 0$, it follows:

$$
p(t)=\mu \cdot P_{k-1}(t)
$$

For the differential equations one finds for $0 \leq t \leq \tau$ :

$$
\begin{aligned}
& P_{0}^{\prime}(t)=-\mu . P_{0}(t) \\
& P_{h}^{\prime}(t)=\mu P_{h-1}(t)-\mu . P_{h}(t) ; \quad 0<h \leq k-1
\end{aligned}
$$

with initial conditions:

$$
P_{0}(0)=1 \quad \text { and } \quad P_{h}(0)=0 ; \quad 0<h \leq k-1 .
$$

The solution is $P_{h}(t)=\mu^{h} t^{h} \mathrm{e}^{-\mu t} / h ! ; 0 \leq h \leq k-1$

$$
W(t)=1-\sum_{i=0}^{k-1} \mu^{i} t^{i} \mathrm{e}^{-\mu t} / i !
$$

For $t>\tau$ the differential equations are:

$$
\begin{aligned}
P_{0}^{\prime}(t) & =-\mu \cdot P_{0}(t)+\eta_{1} \cdot P_{1}(t) \\
P_{h}^{\prime}(t) & =\mu \cdot P_{h-1}(t)-\left(\mu+\eta_{h}\right) \cdot P_{h}(t)+\eta_{h+1} \cdot P_{h+1}(t) ; \quad 0<h<k-1 \\
P_{k-1}^{\prime}(t) & =\mu \cdot P_{k-2}-\left(\mu+\eta_{k-1}\right) \cdot P_{k-1}(t)
\end{aligned}
$$

with initial conditions:

$$
P_{h}(\tau)=\mu^{h} \tau^{h} \mathrm{e}^{-\mu \tau} / h ! \quad 0 \leq h \leq k-1 .
$$

These equations have been solved by conventional methods for $\eta_{h}=h / \tau$ (Ten Hoopen \& Reuver, 1965). 
The result is:

$$
W(t)=1+\sum_{i=1}^{k} \beta_{i} \cdot \exp \left[\alpha_{i}(t-\tau)\right]
$$

$\alpha_{i}$ and $\beta_{i}$ being constants determined by the parameters $\mu, \tau$ and $k$.

\section{Results and Discussion}

In Fig. I values of $(\mu t)_{0.6}$ obtained from the preceding formulae are compared with those from the exact solution for $k=2$ and $k=3$ after Van Elteren \& Gerrits (1961).

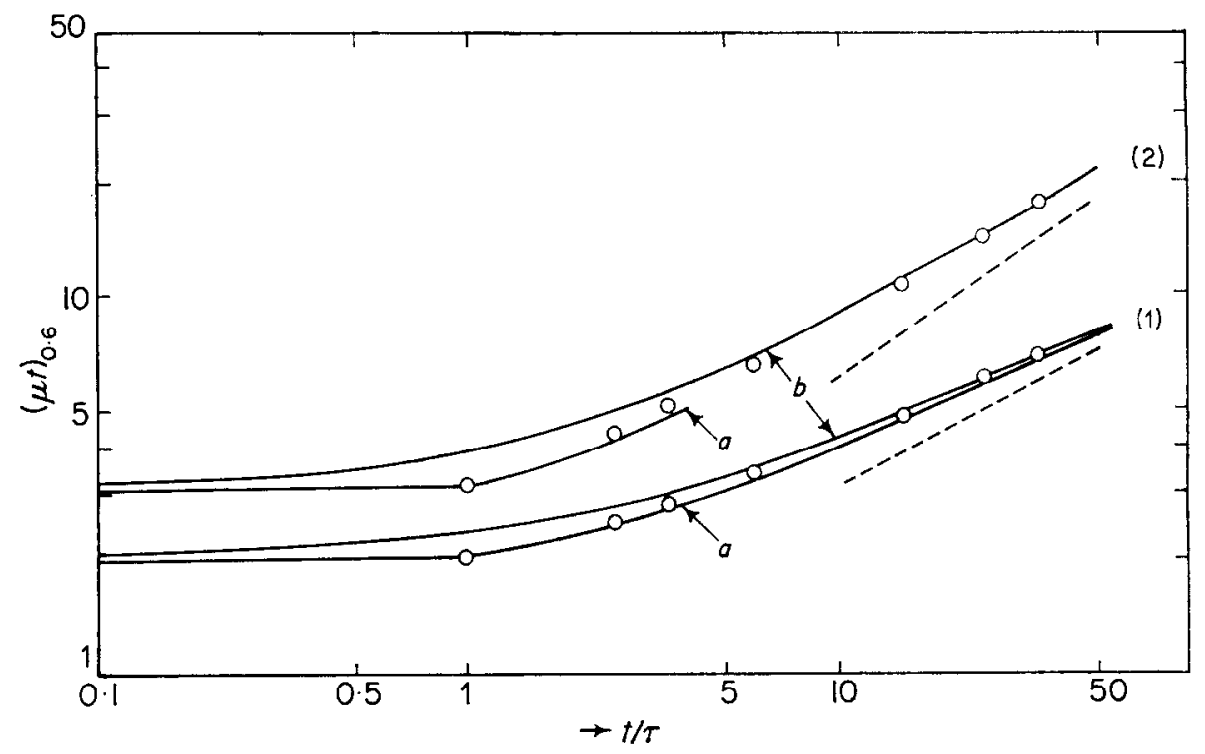

Fig. 1. $(\mu t)_{0 \cdot 6}$ as a function of $t / \tau$ with asymptotes for $k=2(1)$ and $k=3$ (2). Curves (a) from exact solution, circles from approximation; curves $(b)$ from modified model.

The asymptotic behaviour of $(\mu t)_{0 \cdot 6} \approx(t / \tau)^{(k-1) / k}$ for $t \gg \tau$ as stated by Bouman \& Van der Velden (1947) was checked for $k=2$ and $k=3$ and confirmed. This follows from the asymptotic value of $W(t / \tau)$ for $\mu \tau \rightarrow 0$ which is $W(t / \tau) \approx 1-\exp \left(-\mu^{2} \tau^{2} t / \tau\right)$ and $W(t / \tau) \approx 1-\exp \left(-0.5 \mu^{3} \tau^{3} t / \tau\right)$, respectively.

The other extremum $\mu \tau \rightarrow \infty$ has its counterpart in the neurophysiological example. This condition means that the last of a group of $k$ stimuli, impinging after the refractory period is over, produces a response whatever the time relations between them. Then $p(t)$ is given by

$$
p(t)=\mu^{k} t^{k-1} \exp (-\mu t) /(k-1) ! ;
$$


an expression used by Kuffler, FitzHugh \& Barlow (1957) and Bishop, Levick \& William (1964) to characterize the fluctuation in the duration of successive intervals of the maintained discharge of ganglion cells in the cat's retina. Although these authors admit that other formulae might have fitted their data as well, this queuing model can give a justification for applying this distribution by labelling the parameters $k$ and $\mu$ with a physiological meaning.

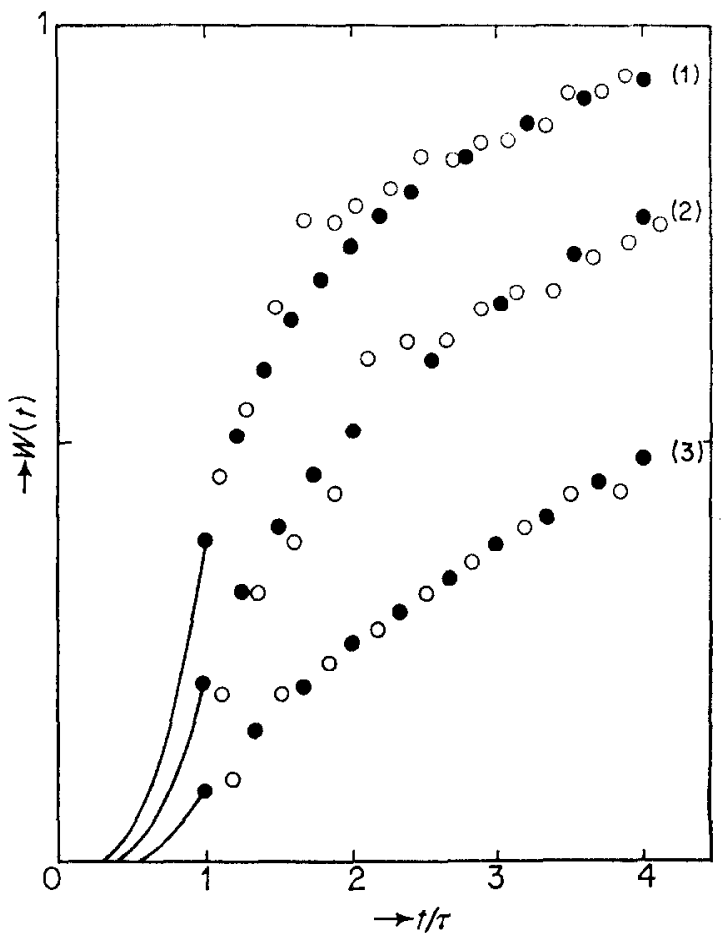

FIG. 2. $W(t / \tau)$ as a function of $t / \tau$ for $k=6 ; \mu \tau=5(1), \mu \tau=4$ (2) and $\mu \tau=3$ (3). Curves for $0<t / \tau \leqslant 1$ from exact solution. For $t / \tau>1$ : circles $(\bullet)$ from approximation; circles ( $O)$ from Monte Carlo simulation ( 50 responses for each series).

In order to obtain an impression of the value of the approximation for higher values of $k$ the results of the formulae for $W(t)$ have been compared with Monte Carlo simulation results in Figs. 2 and 3.

It will be noted that in this approximation the constant holding time $\tau$ is replaced, for $t>\tau$, by an exponentially distributed holding time with parameter $\mathscr{V}$ equal to $1 / \tau$. This, in turn, suggests a modification of the original assumption as to the holding time of the items (quanta and stimuli 
respectively), or in terms of queuing theory, a model with Poisson input and an exponentially distributed holding time with parameter $\mathscr{V}$, having an absorbing barrier at $h=k$ and a reflecting barrier at $h=0 . W(t)$ and $p(t)$ for this model can be solved exactly. The differential equations for this case are the same as above for $t>\tau$ when $\eta_{h}$ is replaced by $\mathscr{V} . h$ and with initial conditions: $P_{0}(0)=1$ and $P_{h}(0)=0$ for $0<h \leq k-1$.

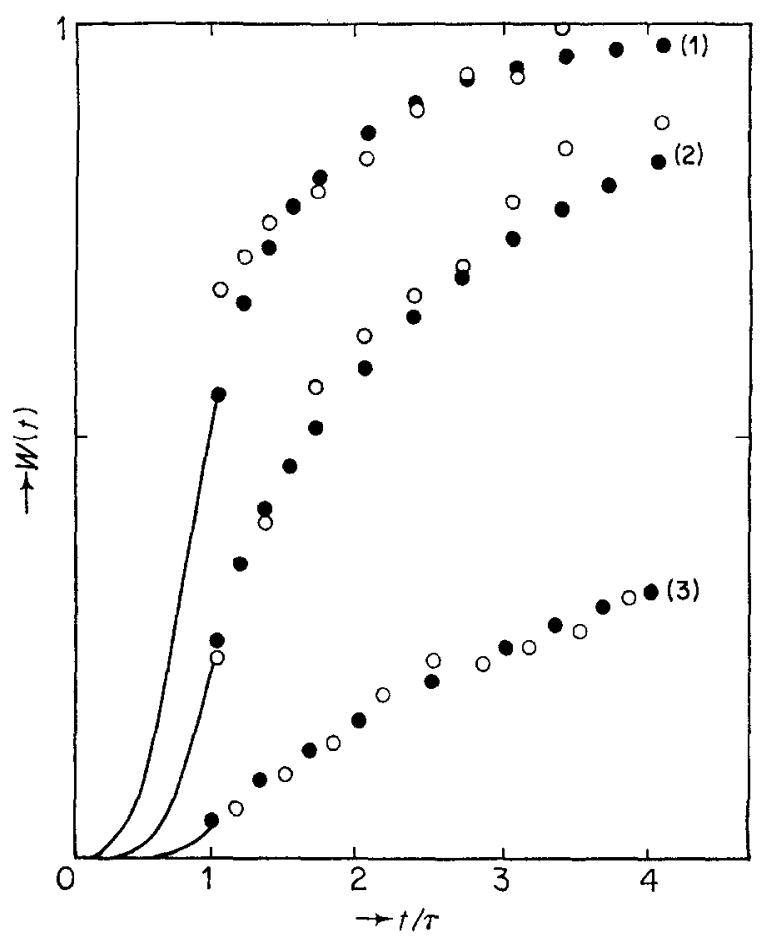

FIG. 3. As Fig. 2 for $\mu \tau=6 ; k=6(1), k=8$ (2) and $k=11$ (3).

In both the case of the light quanta and the nervous impulses such a proposition needs not, a priori, be less realistic than that of a constant holding time.

For the $(\mu t)_{0.6}$ vs. $t / \tau$ relation this model has been worked out for $k=2$ and $k=3$ in Fig. 1 (curves $b$ ). These curves are more smooth than those for a constant holding time, the asymptotes for $t / \tau \rightarrow 0$ and $t / \tau \rightarrow \infty$ being the same. At present the experimental data reported in the literature are too scarce to decide which of the two models is to be preferred. 


\section{REFERENCES}

Bishop, P. O., LeVick, W. R. \& William, W. O. (1964). J. Physiol. 170, 598.

Bouman, M. A. \& Van der Velden, H. A. (1947). J. opt. Soc. Amer. 37, 908.

ECCLEs, J. C. (1953). “The Neurophysiological Basis of Mind". London: Oxford University Press.

Van Elteren, Ph. \& Gerrits, H. J. M. (1961). Statist. Neerl. 15, 385.

Kuffler, S. W., FitzHugh, R. \& Barlow, H. B. (1957). J. gen. Physiol. 40, 683.

Ten Hoopen, M. \& Reuver, H. A. (1965). Statist. Neerl. In press.

RAPOPORT, A. (1950). Bull. math. Biophys. $12,187$.

SHOLL, D. A. (1956). "The Organization of the Cerebral Cortex". London: Methuen.

VAN Der Velden, H. A. (1944). Physica, Eindhoven, 11, 179. 